\title{
Evaluations at the University of Natural Resources and Applied Life Sciences Vienna (BOKU)
}

The evaluations which have been carried out at BOKU during the last years have to be seen embedded into the university-wide quality management system which aims at comprising all quality relevant areas, further developing evaluation procedures and QA instruments, optimizing organisational and decision making processes and building up a quality culture. It can be summarised as follows:

\section{Framework:}

University Act 2002, Intellectual Capital Report, ENQA-Standards

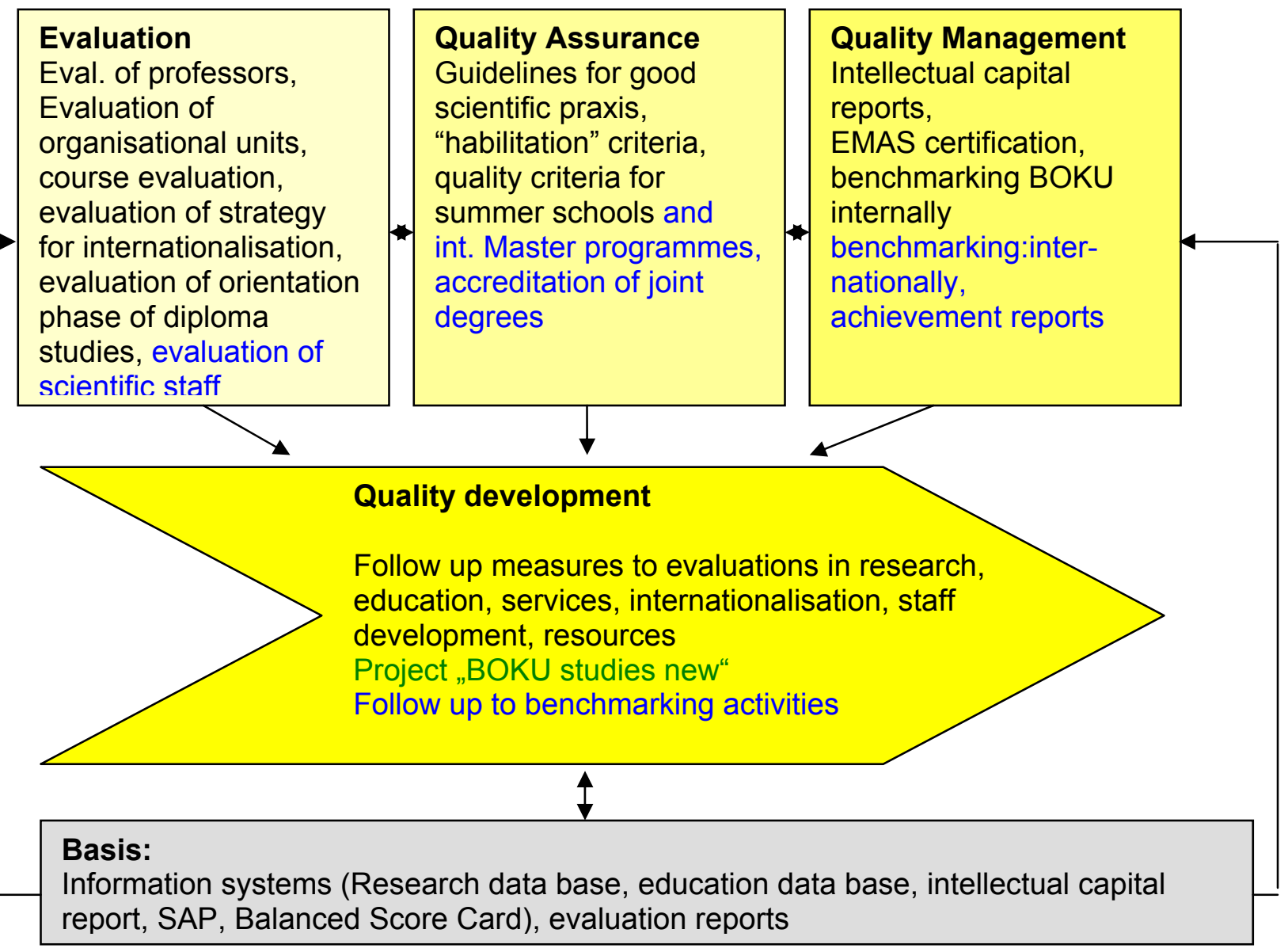

black: realized and/or continued

green: $\quad$ ongoing

blue: $\quad$ planned 
In the following some of the major evaluations are listed which had been carried out at BOKU or in cooperation with BOKU.

\section{Evaluation of organisational units}

BOKU plans to evaluate all organisational units from 2006 onwards in a cyclical manner so that within two performance agreement periods (6 years) all departments shall be evaluated. In 2005, the "Guidelines for evaluating organizational units according to the University Act of 2002"1 have been elaborated taking into account the standards of the German Society for Evaluation (DeGEval) of which BOKU is an institutional member and the guidelines of the European Network for Quality Assurance (ENQA).

The core of this evaluation is a peer review which is combined with quality assurance and quality management elements in order to raise objectivity, transparency and sustainability. In reference to $Q A$ an internal benchmarking activity is been carried out; the most important core achievement indicators of an evaluated department are anonymously compared with those of all other departments. In reference to the latter, the self evaluation questionnaire is strictly based on the QM steps and all core achievement processes have to be described and analysed according to this concept.

In 2006 the department for biotechnology is evaluated, the findings will directly result into the internal performance agreement between the department and the rectorate for the following year.

In 2004, when the BOKU structure was changed from 41 institutes to 13 departments an evaluation of this new structure was carried out on the basis of a peer review. Its results were incorporated into the first internal performance agreement between the rectorate and the departments.

\section{Course evaluations}

BOKU was the first Austrian university to implement course evaluations in 1996 and in 2005 this concept was thoroughly reviewed. On the one hand, criteria and questions for all types of courses, on the other hand also specific criteria for several courses (e.g. seminars, practical courses and excursions) were developed and combined into one questionnaire.

Another change was that the students were not only asked at the end of the semester for their feedback, but also during the semester so that the teachers got the chance to carry out improvements also during the realisation of a course. Finally there was also a change from a paper based evaluation to an electronic version which was integrated into the education information system called BLIS++. ${ }^{2}$

\section{Evaluations of professors}

During the last five years new professors at BOKU were only given temporary contracts which ranged from three to seven years. Before a contract terminates an evaluation is carried out in order to find out to which extent the candidate had met the agreed objectives. Following a standardized procedure which is guided by a scientific commission, the candidate first fills in a self evaluation report which is reviewed by two

\footnotetext{
${ }^{1}$ See: http://www.boku.ac.at/7509.html?\&L=1\%2522

2 See: http://blis.ud.boku.ac.at/ 
external international experts. On the basis of these two expert opinions the commission elaborates a final report and a recommendation to the rector who finally decides about the prolongation of the contract of the candidate. The results of these evaluations help to set the basis for future contracts.

In the course of the six evaluations of professors which had been carried out in 2005 and 2006 the evaluation concept has been further improved.

\section{Evaluation of the new Bachelor and Master curricula}

BOKU was the first university in Austria to switch completely from the old diploma to the new two/three cycle study structure.

First a comprehensive situation analysis of partners and competitors on national and international level was carried out. Then benchmarking activities were undertaken and various experts in the field, especially form the industry, personnel managers and representatives from the Ministry of Education, Science and Culture were interviewed in order to get information on present and possible future fields of work.

This was a good basis for the elaboration of a self report through the study committees. Consequently workshops were held which led to the draft of a new curriculum. In order to obtain an additional view from external experts a peer review was carried out. The international peers provided support for the design of the new curricula in terms of content, didactics, structure and organisation. Most of the recommendations could be considered and led to the final version of the new study programmes which started in October 2003.

\section{Evaluation of the implementation of the Bologna reform}

BOKU participated together with 8 other European universities in the third round of the Quality Culture project of the European University Association (EUA) which aimed at developing "good practices for developing high quality study programmes and implementing them in a coherent way".

The core of this project was an evaluation on basis of a SWOT analysis for which all stakeholders of curriculum development and programme delivery were considered. 13 qualitative interviews were carried which were discussed in an expert workshop. The action plan which was elaborated afterwards comprised the following main objectives:

1. Development, redesign and consolidation of study programmes at BOKU

2a. Quality assurance of the level of study programmes at BOKU

2b. Quality assurance of the level of BOKU courses

3. Maintenance of the high importance and prestige of education at BOKU

4. Improvement of data management and information systems in the area of education

In order to implement the outcomes of this evaluation the senate started a three years project called „BOKU studies new“. 


\section{Evaluation of summer schools and international degree programmes}

The Euro League of Life Sciences (ELLS) ${ }^{3}$ is a quality network of 7 European universities in the Life Sciences which aims at developing and delivering high quality study programmes and courses. Within a pilot project last year an evaluation procedure for international summer schools was elaborated (mid term and final evaluation, further QA measures). This year, six summer schools were evaluated on the basis of this procedure.

The quality assurance working group within ELLS which is co-ordinated by BOKU is currently working on quality criteria and benchmarks for evaluating international Master programmes. This group also works on finding ways how to accredit joint and double degree programmes. In this area it also cooperates with the Interuniversity Consortium for Agricultural Sciences and Related Sciences (ICA $)^{4}$ which coordinates two projects of the European Commission in this area.

Thomas Guggenberger, 31. 10. 2006

\footnotetext{
${ }^{3}$ See: http://www.euroleague-study.org/index.html

${ }^{4}$ See: http://www.ica-europe.infol
} 